\title{
TEMPERATURE DEPENDENT ELECTRON BEAM INDUCED CURRENT STUDY OF DEFECTS IN SILICON
}

\author{
T. Sekiguchi, S. Kusanagi, Y. Mryamura* and K. Sumino \\ Institute for Materials Research, Tohoku University, Sendai 980, Japan \\ Dedicated to Professor Dr. Julian Auleytner \\ on the occasion of his 70th birthday
}

(Received November 23, 1992)

\begin{abstract}
A new computer-aided electron beam induced current system was developed which makes it possible to obtain two-dimensional mapping of the absolute magnitudes of electron beam induced current signals over the temperature range $15 \mathrm{~K}-400 \mathrm{~K}$. Electronic states of defects in cast silicon and deformation-induced dislocations in float-zone silicon were investigated from the analyses of temperature dependencies of electron beam induced current contrasts of the defects measured with the system. Electron beam induced current active defects in cast Si were identified to be Fe impurity atoms or $\mathrm{Fe}-\mathrm{B}$ pairs incorporated at the dislocation core depending on the cooling rate of a crystal. Dislocations in float-zone silicon were shown to have an energy level for carrier recombination in the lower half of the band gap.
\end{abstract}

PACS numbers: 71.55.Fr, 72.80.Cw

\section{Introduction}

Electron beam induced current (EBIC) imaging is one of the most convenient techniques to reveal spatial distribution of electrically active defects in semiconductors. However, the conventional EBIC technique has, at least, two weak points in clarifying the physics of defects. One is the lack of the quantitativeness of data resulting from instrumentation which uses photofilms in recording defect images. Recording with a photofilm does not give the absolute magnitudes of EBIC signals despite that it has advantage of high spatial resolution. The other weak point is that any defect which acts as a recombination center gives similar EBIC contrast irrespective of its energy level. Hence, it is impossible to distinguish different kinds of defects from each other from their EBIC images alone.

*Present address: Research Laboratory, Komatsu Electronic Metals Co. Ltd., Hiratsuka 254, Japan. 
To overcome the first weak point, we developed a computer-aided quantitative EBIC system which records absolute magnitudes of EBIC signal from a specimen in two-dimensional array. The block diagram of our EBIC system is shown in Fig. 1. The whole system is controlled by a microcomputer and the electron beam

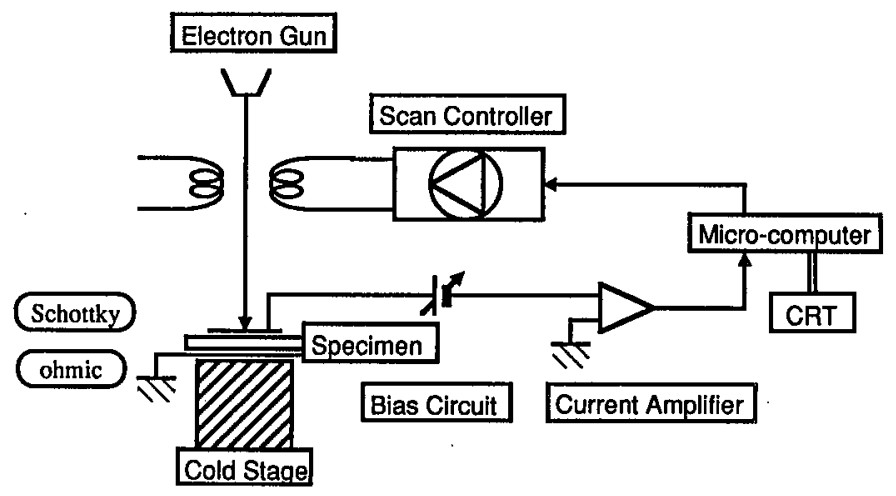

Fig. 1. Block diagram of the new EBIC system.

scans the specimen surface dividing the area under observation into $320 \times 320$ dots. The EBIC signals are stored digitally within a memory with a resolution of 12 bits. One EBIC image is normally recorded into 200 kilobytes memory within two minutes. Image processing such as filtering, output of differential or intensity profile as well as the calculation of the contrast of any defect can easily be done with the use of a software. In addition, other kinds of responses to the electron beam excitation, such as emission of secondary electrons or reflected electrons, cathodoluminescence, etc., of the same area can also be recorded. Combining all the kinds of responses, we are able to deduce various aspects of the nature of the same defect.

The second weak point can be overcome by utilizing the fact that, in general, different kinds of defects show different dependencies of recombination activities on experimental parameters such as temperature, excitation condition, etc. For instance, change in temperature results in the change in the rate of electronic process depending on the concerned energy level. Therefore, from the analysis of temperature dependence of the EBIC contrast, we can distinguish defects having different levels for recombination process. On the basis of such idea, we introduced a cooling stage into our system which makes it possible to measure the temperature dependence of EBIC signal. The stage was designed in such a way that the specimen temperature is controlled in the range between $15 \mathrm{~K}$ and $400 \mathrm{~K}$ using liquid helium as the coolant.

In this paper we report results of temperature dependent EBIC examinations of defects in polycrystalline cast silicon and that of dislocations in float-zone (FZ) silicon crystals generated from scratches under stress. 


\section{Experimental}

Polycrystalline cast silicon was $p$-type doped with B prepared by casting melts into carbon crucibles. The carrier concentration was approximately $1 \times 10^{16} \mathrm{~cm}^{-3}$. It contains much higher densities of impurities and defects both in species and concentration than Czochralski (CZ) or float-zone (FZ) silicon crystals. The specimens were sealed into quartz capsules in vacuum and annealed at elevated temperature for a certain period and cooled at various rates. Rapid cooling of a specimen from the annealing temperature was realized by taking out the capsule from the furnace and breaking it in ice-water. The cooling rate was estimated to be higher than $1000^{\circ} \mathrm{C} / \mathrm{s}$. Slow cooling of a specimen was done by means of furnace cooling at a rate of $100^{\circ} \mathrm{C} / \mathrm{h}$. The detailed procedure was reported in a previous paper [1, 2].

As for the study of dislocations in FZ silicon, specimens of both $p$ - and $n$-types were prepared. Dopants were $\mathrm{B}$ in $p$-type specimens and $\mathrm{P}$ in $\boldsymbol{n}$-type specimens. The carrier concentrations of both types of specimens were approximately $1 \times 10^{16} \mathrm{~cm}^{-3}$. Fresh dislocations were introduced from scratches drawn along the [110] direction on the (111) surface by means of three-point bending at $700^{\circ} \mathrm{C}$ in a vacuum. The geometry of the specimen is given in Fig. $2.60^{\circ}$ parts of dislocation

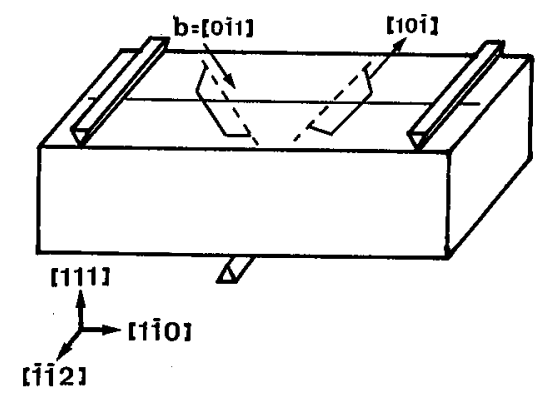

Fig. 2. Geometry of specimen for the study of dislocations in FZ Si.

half-loops emerge on the (111) surface and are observed by means of both the etch pit and EBIC technique.

After treatment mentioned above the Schottky contact was fabricated on the (111) specimen surface with evaporation of $\mathrm{Au}$ in the case of an $n$-type specimen and $\mathrm{Al}$ in the case of a p-type specimen. EBIC observations were carried out under electron beam excitation with an accelerating voltage of $20 \mathrm{kV}$ and a beam current of $2 \times 10^{-10} \mathrm{~A}$ under zero bias. The specimen temperature was varied from $40 \mathrm{~K}$ to $350 \mathrm{~K}$.

The EBIC contrast $C$ of a defect is defined by

$$
C=\left(I_{\mathrm{b}}-I_{\mathrm{d}}\right) / I_{\mathrm{b}}
$$

where $I_{\mathrm{b}}$ and $I_{\mathrm{d}}$ are EBIC currents of the background and at the defect, respectively. 


\section{Results and discussion}

\subsection{Defects in cast silicon}

Figure 3 shows EBIC images at $300 \mathrm{~K}$ (left) and at $60 \mathrm{~K}$ (right) of the cast Si after slow cooling (top) and after rapid cooling (bottom) from $800^{\circ} \mathrm{C}$, respectively. Dark spots are seen in a rapidly cooled specimen both at $300 \mathrm{~K}$ and $60 \mathrm{~K}$. In a
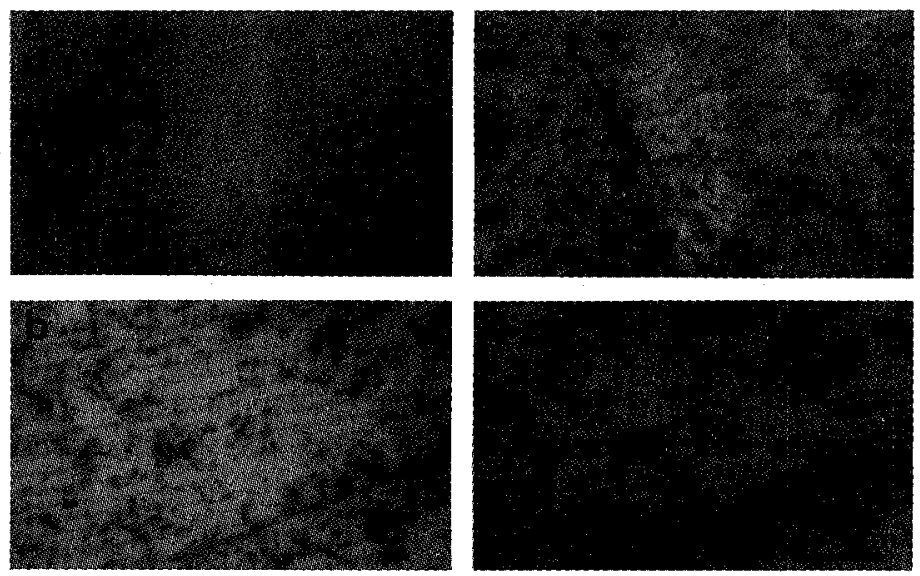

Fig. 3. EBIC images of cast Si slowly cooled (a, c) and rapidly cooled (b, d) from $800^{\circ} \mathrm{C}$, taken at $300 \mathrm{~K}(\mathrm{a}, \mathrm{b})$ and $60 \mathrm{~K}(\mathrm{c}, \mathrm{d})$.

slowly cooled specimen, on the other hand, the EBIC image does not show any contrast at $300 \mathrm{~K}$, while dark spots are visible at $60 \mathrm{~K}$. The dark spots are about 3 micron in the diameter and about $10 \%$ in the contrast in the rapidly cooled specimen observed at $300 \mathrm{~K}$. These spots were all found to have correspondence to dislocations revealed by the etch pit technique.

In the rapidly cooled specimen dark spots are visible at $300 \mathrm{~K}$ when it is cooled from temperatures higher than $600^{\circ} \mathrm{C}$ but not when cooled from lower temperatures. Both the contrast and the density of dark spots increase as annealing temperature increases but do not depend on the duration of annealing. Some dislocation etch pits have no correspondence to dark spots when the specimen is cooled from low temperatures. The appearance of the dark spots is determined only by rapid cooling from high temperature just prior to the observation and is not influenced by thermal history of the specimen. Such characteristic of the dark spots is quite similar to that of quenched-in defects in FZ or CZ Si reported previously [1].

In the slowly cooled specimen dark spots in EBIC image are seen when the temperature of observation is lower than $150 \mathrm{~K}$ but not at higher temperatures. This implies that the defects giving rise to the dark dots in such specimens are electrically inactive in the temperature range higher than $150 \mathrm{~K}$.

We may conclude that defects giving rise to the dark spots in EBIC image are different in electrical activities between the rapidly cooled specimen and the slowly 
cooled specimen. It is conceivable that defect reaction which takes place within the specimen during cooling from high temperature is influenced by cooling rate.

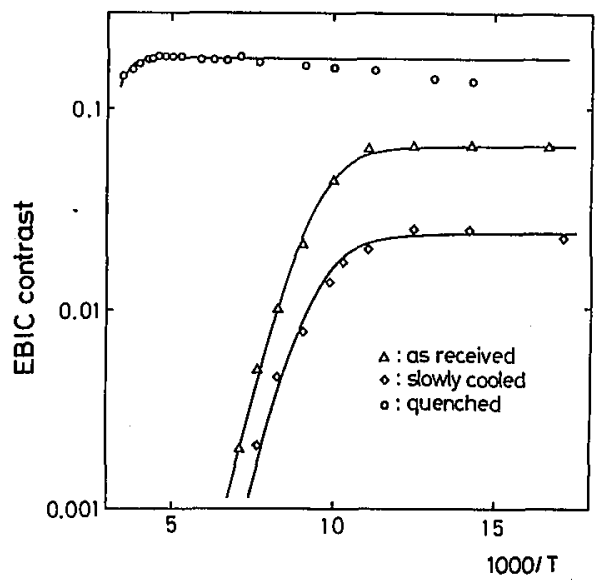

Fig. 4. Temperature dependencies of EBIC contrasts of defects in rapidly cooled, slowly cooled and as received specimens of cast $\mathrm{Si}$.

Figure 4 shows the EBIC contrasts of dark spots in a rapidly cooled specimen and a slowly cooled specimen measured against inverse temperature $1 / T$. The data for a specimen prepared from an as received crystal without any heat treatment is also shown for the comparison's sake. The temperature dependence of the EBIC contrast in as received specimen is very similar to that in the slowly cooled specimen.

We assume that the recombination rate at the defect is controlled by the occupation probability of the defect level which is determined by the temperature. We further assume that the concerned defect accompanies only one energy level and that its EBIC contrast is proportional to the occupation probability of the level. We apply the Shockley-Read-Hall statistics under electron irradiation. Using the idea of pseudo-Fermi level, the EBIC contrast of the defect at temperature $T$ is deduced to be

$$
C(T) / C(0)=1 /\left[1+A T^{3 / 2} \exp \left(-E_{\mathrm{D}} / k T\right)\right],
$$

where $C(0)$ is the EBIC contrast at absolute zero, $E_{\mathrm{D}}$ is the energy level of the defect, $A=C_{\mathrm{v}} / p$, where $C_{\mathrm{v}}$ and $p$ are the effective state density of valence band and the hole concentration under electron beam excitation, respectively, and $k$ is the Boltzmann constant.

From the fitting of the curves in Fig. 4 with Eq. (2) the energy level $E_{\mathrm{D}}$ of the EBIC-active defects in cast silicon is determined to be $E_{\mathrm{v}}+0.11 \mathrm{eV}$ for both the slowly cooled specimen and the specimen in the as grown state, while $E_{\mathrm{v}}+0.37 \mathrm{eV}$ for the rapidly cooled specimen. These values are approximately equal to the energy levels of a $\mathrm{Fe}-\mathrm{B}$ pair and an interstitial $\mathrm{Fe}$ atom in silicon, respectively. Therefore, we may attribute the EBIC-active defects to Fe impurity which is incorporated at the dislocation core on the assumption that electronic 
states of both an interstitial $\mathrm{Fe}$ atom and a $\mathrm{Fe}-\mathrm{B}$ pair located at the dislocation core are the same as those in the matrix Si crystal. Fe atoms are thought to be distributed individually along the dislocation line in the rapidly cooled specimen while they combine with B atoms in the slowly cooled specimen. Fe impurity is thought to be incorporated into the Si crystals at the time of casting.

\subsection{Dislocation in FZ Si}

Figures $5 \mathrm{a}$ and $5 \mathrm{~b}$ show etch pits and EBIC images at $300 \mathrm{~K}$, respectively, of dislocations which were generated from a scratch on the (111) surface of a specimen of FZ Si.
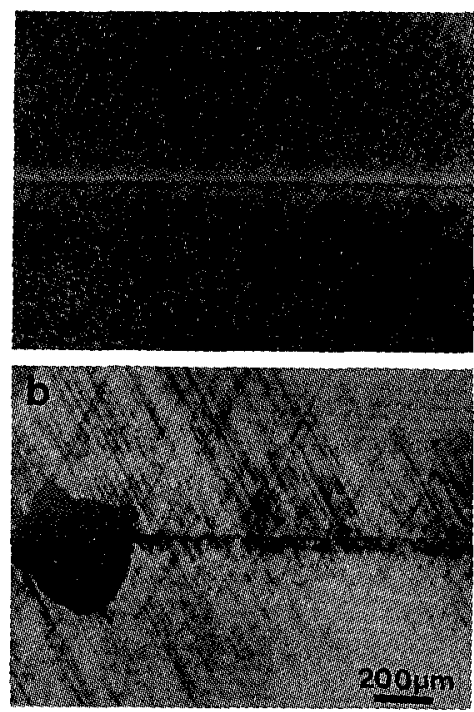

Fig. 5. (a) Etch pits and (b) EBIC images of dislocations in $p$-type FZ Si.

Figure 6 shows the temperature dependencies of EBIC contrast of a $60^{\circ}$ dislocation in $p$-type and $n$-type FZ Si. In $p$-type Si the EBIC contrast increases rapidly with increasing temperature in a low temperature range, attains the maximum at about $100 \mathrm{~K}$, and then decreases gradually up to $320 \mathrm{~K}$. On the other hand, in $n$-type Si the EBIC contrast increases rapidly with increasing temperature and attains a plateau without showing the maximum.

The model applied to the temperature dependence of the EBIC contrast mentioned in the previous section does not give a correct description for that shown in Fig. 6. The increase in the EBIC contrast with temperature suggests that the trapping rate of carriers at the defect level increases with temperature. This may be realized if the defect accompanies some potential barrier for carrier trapping: namely, carriers are trapped at the defect by means of thermal activation. Such potential barrier may be caused by the Coulomb potential of a charged dislocation or large lattice relaxation at the dislocation core. The decrease in the contrast in the high temperature range in $p$-type silicon which is absent in $n$-type silicon can 


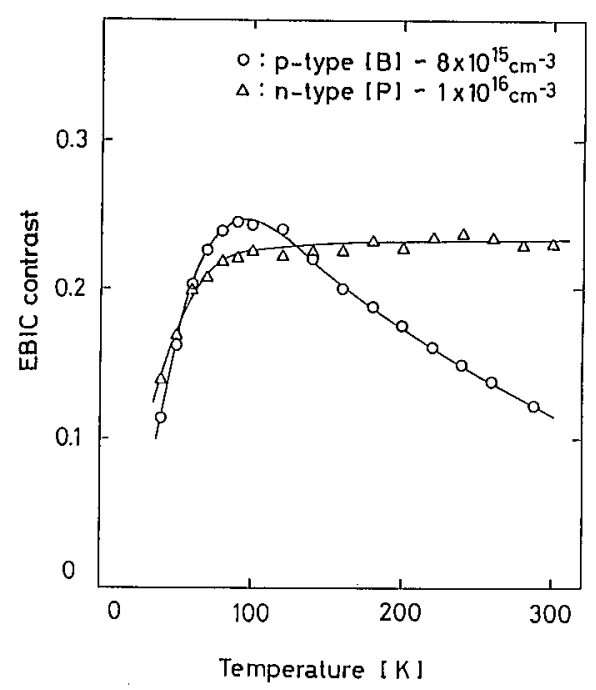

Fig. 6. Temperature dependencies of EBIC contrasts of dislocations in $n$-type and $p$-type FZ Si.

be explained if the defect level is located closer to the valence band than to the conduction band.

Wilshaw et al. [3] discussed the temperature dependence of the EBIC contrast of a dislocation on the basis of the model that the Coulombic potential developed around a charged dislocation line which gave rise to the band bending around the dislocation. Fitting of the EBIC data in Fig. 6 to his model gives the energy level of the defect at $E_{\mathrm{v}}+0.2 \mathrm{eV}$ and the line density of the defects to be $2 \times 10^{6} \mathrm{~cm}^{-1}$ along the dislocation line. It is not clear at present how such a value of line density of the defects is related to the realistic picture of electronic state of a dislocation.

Figure 7 compares EBIC contrasts of dislocations in a $p$-type specimen before and after heat treatment at $800^{\circ} \mathrm{C}$ for $1 \mathrm{~h}$. The dislocation contrast after annealing is essentially the same as that before annealing in the temperature dependence, though the absolute value of contrast shows some decrease due to annealing. It suggests that EBIC activity of a dislocation is not altered by annealing at $800^{\circ} \mathrm{C}$.

The electronic state of a dislocation in a semiconductor has long been an object of dispute. Investigations by means of deep level transient spectroscopy (DLTS) [4-6] and ESR [7, 8] have revealed that several deep levels are induced in $\mathrm{Si}$ by plastic deformation. However, since such deep levels are eliminated by annealing at temperatures higher than $800^{\circ} \mathrm{C}$, it is now accepted that they are mostly attributed to point defects or their clusters which are introduced during deformation and that the regular parts of dislocations are electrically inactive since geometrical dangling bonds along the dislocation core are reconstructed. Only some disturbed parts of the dislocation line such as reconstruction defects (solitons), kinks, and jogs are believed to accompany deep electronic levels. 


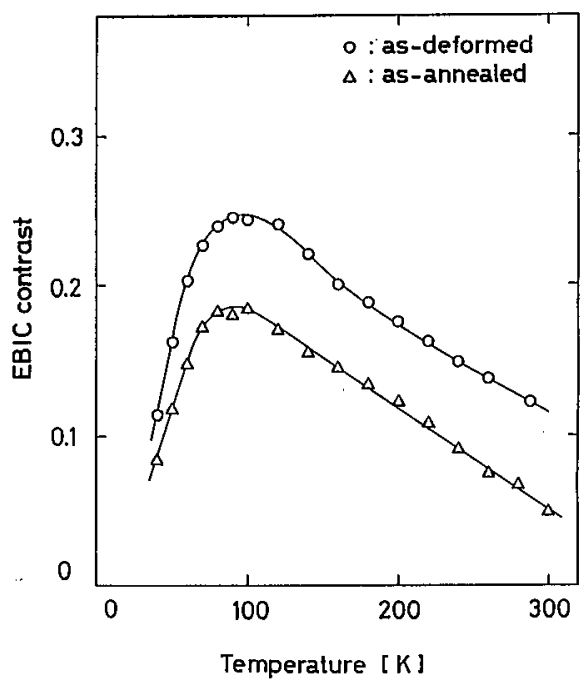

Fig. 7. Temperature dependencies of EBIC contrasts of dislocations in $p$-type Si in as deformed state and after annealing at $800^{\circ} \mathrm{C}$.

On the other hand, it has been known that dislocations give rise to EBIC contrasts [3,9-11]. This point has been confirmed also in the present work. The present work has also shown that the EBIC contrast related to dislocations survives after annealing at $800^{\circ} \mathrm{C}$ by which all hole traps detected by DLTS or ESR-active centers have been reported to be eliminated. This seems to mean that the EBIC contrasts observed in the present work are really related to dislocations themselves. In other words, dislocations act as recombination centers of carriers. It is probable that only some special parts of a dislocation line are active as recombination centers. They seem to be distributed along the dislocation line rather uniformly with some separation to give rise to a continuous EBIC contrast along the line [11]. The volume density of such parts may be too low to be detected with usual DLTS or ESR technique.

Plastic deformation of an $\mathrm{Si}$ crystal is known to induce characteristic photoluminescence lines termed $D 1$ through $D 4$ which survive after annealing which eliminates deformation-induced deep levels detected by DLTS or ESR [12-15]. Recently, these photoluminescence lines are related to some metallic contamination of dislocations [16]. It is interesting to investigate whether the photon energies of luminescence lines are related to the energy level giving rise to the EBIC contrast of dislocations. Such a work is now under progress.

Kusanagi et al. recently observed EBIC images of dislocation loops lying on slip planes parallel to the specimen surface in $n$-type $\mathrm{Si}$ [11]. They found that the EBIC activities of the $60^{\circ}$ parts and the screw parts of a dislocation loop are different from each other from different temperature dependencies of the EBIC contrast. The temperature dependence of the EBIC contrast of the $60^{\circ}$ part was observed to be different from the results of the present work. Such difference may originate 
from the difference in excitation level and/or the difference in the contamination level of dislocations between the two works.

\section{Conclusion}

A new computer-aided EBIC system was developed. Quantitative EBIC studies of defects in cast $\mathrm{Si}$ and dislocations in $\mathrm{FZ} \mathrm{Si}$ as functions of temperature have been accomplished with this system. Analyses of the data show that the energy levels of defects in cast $\mathrm{Si}$ is $0.37 \mathrm{eV}$ or $0.11 \mathrm{eV}$ above the valence band, depending on the cooling rate of the crystal, and that the energy level of $60^{\circ}$ dislocations for carrier recombination is located in the lower half of the band gap.

\section{References}

[1] T. Iwasaki, T. Sekiguchi, Y. Miyamura, K. Sumino, in: Defect Control in Semiconductors, Vol. 1, Ed. K. Sumino, North-Holland, Amsterdam 1990, p. 365.

[2] T. Sekiguchi, Y. Miyamura, K. Sumino, J. Phys. (France) 24, C6-168 (1989).

[3] P.R. Wilshaw, T.S. Fell, G.R. Booker, in: Point and Extended Defects in Semiconductors, NATO ASI Series B, Vol. 202, Eds. G. Benedek, A. Cavallini, W. Schroeter, Plenum Press, New York 1989, p. 243.

[4] J.R. Pattel, L.C. Kimmering, Cryst. Res. Technol. 16, 187 (1981).

[5] V.V. Kveder, Yu.A. Osip'yan, W. Schroeter, G. Zoth, Phys. Status Solidi A 72, 701 (1982).

[6] H. Ono, K. Sumino, J. Appl. Phys. 57, 287 (1985).

[7] E.R. Weber, H. Alexander, J. Phys. (France) 40, C6-101 (1979).

[8] M. Suezawa, K. Sumino, K. Iwaizumi, J. Appl. Phys. 54, 6594 (1983).

[9] A. Ourmazd, Cryst. Res. Technol. 16, 173 (1981).

[10] C. Donolato, J. Phys. Colloque 44, C4-269 (1983).

[11] S. Kusanagi, T. Sekiguchi, K. Sumino, Appl. Phys. Lett. 61, 792 (1992).

[12] N.S. Drozdov, A.A. Partin, V.D. Tkachev, Sov. Phys.-JETP Lett. 23, 597 (1976).

[13] M. Suezawa, K. Sumino, Phys. Status Solidi A 78, 639 (1983).

[14] M. Suezawa, Y. Sasaki, K. Sumino, Phys. Status Solidi A 79, 173 (1983).

[15] R. Sauer, J. Weber, J. Stolz, E.R. Weber, K.H. Kuesters, H. Alexander, Appl. Phys. A 36, 1 (1985).

[16] V. Higgs, E.C. Lightowlers, P.C. Kightley, in: Mater, Res. Soc. Symp. Proc., Vol. 163, Eds. D.J. Wolford, J. Bernholc, E.E. Haller, Mater. Res. Soc., Pittsburgh 1990, p. 57. 